Pramāna, Vol. 11, No. 3, September 1978, pp. 245-250, (C) printed in India

\title{
Correlation of acoustic and thermal anomalies in ferroelectric crystals
}

\author{
S K KHANNA and P S NARAYANAN \\ Department of Physics, Indian Institute of Science, Bangalore 560012 \\ MS received 9 March 1978
}

Abstract. The Pippard-Janovec relations are derived for correlating the anomalous elastic coefficient and the anomalous specific heat near the phase transitions of ferroelectric crystals. These relations are verified in the case of ferroelectric triglycine selenate crystal.

Keywords. Pippard-Janovec relations; ultrasonic velocity; ferroelectrics, triglycine selenate.

\section{Introduction}

The ultrasonic investigation of thermodynamic systems such as ferroelectric crystals near their phase transitions are intensively studied in recent times. One of the advantages of such a study is that one can investigate simultaneously the static and the dynamic properties of the system (Garland 1970). It is very important, therefore to look for the methods of verifying the consistency of ultrasonic data. In the present paper we develop a relation between the elastic constants and the specific heat at constant pressure which change anomalously near the phase transitions in ferroelectric crystals. Such a relation is necessary in order to correlate the acoustic and thermal anomalies.

\section{Formulation of the problem}

It is well known that various thermodynamic quantitites change discontinuously near the phase transition of first order. The slope of the transition curve could be related with those discontinuous changes by Clasius-Clapeyron equation. A similar relation was derived by explaining the behaviour of various thermodynamic quantities like specific heat at constant pressure, isobaric thermal expansion coefficient, isothermal compressibility which experience finite changes near the second order phase transition. With the studies on $\lambda$-transition in liquid ${ }^{4} \mathrm{He}$ it has been realised that Ehrnfest's relations for the second order phase transition break down in instances where $C_{p}, k_{T}, a$ show logarithmic or other singularities at the transition temperature.

The entropy, which is a function of temperature and pressure, rises steeply to a vertical or nearly vertical tangent along the $\lambda$-line and the analytical form of the entropy surface is dominated by this circumstance. Pippard $(1956,1957)$ was the 
first to say that in the neighbourhood of the $\lambda$-line the surface may be regarded as cylindrical in the sense that the only principal curvature of significance is that in plane sections normal to $\lambda$-line. With this approximation Pippard was able to overcome the difficulty faced by Ehrnfest's relations.

Using this approximation Pippard derived the following relations (Buckingham and Fairbank 1961) connecting the anomalous quantities near $\lambda$-transition.

$$
\begin{aligned}
C_{p} / T & =(d P / d T)_{\lambda} a V+(d S / d T)_{t} \\
& =(d P / d T)_{\lambda} k_{T}+(1 / V)(d V / d T)_{t}
\end{aligned}
$$

where $(d S / d T)_{t}$ and $(1 / V)(d V / d T)_{t}$ are slowly varying quantities and can be considered as constants.

Both these equations have been verified at the $\lambda$-transitions in liquid $\mathrm{He}, \mathrm{NH}_{4} \mathrm{Cl}$, quartz and a number of ferromagnetic and anti-ferromagnetic transitions (Pippard 1956, Hughes and Lawson 1962).

Garland (1964) developed the generalized Pippard equations in terms of stressstrain variables rather than pressure-volume variables. These relations have been verified on quartz near $a-\beta$ transition.

Janovec (1966) extended the treatment of Buckingham and Fiarbank (1961) for the $\lambda$-transitions and applied it to anisotropic dielectrics. General thermodynamic relations obtained in this way are used to find the coupling between various anomalous quantities near the transition.

The relations obtained by Janovec connects the elastic compliance with the various thermodynamic quantities.

$$
s_{\mu \nu}^{T_{i} E}=-\left(\frac{\partial T}{\partial \sigma_{\mu}}\right) a_{\mu}^{\sigma, E}+s_{\mu \nu}^{t, E}
$$

where $s_{\mu \nu}$ is the elastic compliance and $\alpha_{\mu}$ is the coefficient of linear expansion.

It would be desirable to obtain a relation which would connect the various thermodynamic quantities with the elastic coefficient since from ultrasonic velocity data the quantity which we directly calculate are the elastic coefficients.

\section{Derivation and result}

It is possible to describe a thermodynamic system by suitable independent variables, each being chosen from the pairs of variables $(T, S),(X, x)$ and $(E, P)$. The conventional choice of independent variables is $\sigma$ along with $T$ and $E$ (intensive quantities). However, in the present study, as will be seen in the later section, it was found convenient to derive relations treating $u$ as the independent variable and take into account the relation between $C^{u, E}$ and $C^{\sigma, E}$ before comparison with experimental data.

Let the dielectric crystal at temperature $T$ be under the effect of electric field $\bar{E}$ and mechanical stress $\sigma$ and we take $T, u, E$ as independent variables and $S, \sigma, P$ the dependent variables where $u$ and $P$ are the mechanical strain and the electric 
polarisation. The partial derivatives of dependent variables with respect to independent constitute the matrix

$$
\left[\begin{array}{rrr}
\frac{C}{T}=\frac{\partial S}{\partial T} & \frac{\partial S}{\partial u_{t}} & \frac{\partial S}{\partial E_{i}} \\
\frac{\partial \sigma_{i}}{\partial T} & \lambda_{i j}=\frac{\partial \sigma_{i}}{\partial u_{j}} & \frac{\partial \sigma_{i}}{\partial E_{j}} \\
p_{i}=\frac{\partial P_{t}}{\partial T} & \frac{\partial P_{t}}{\partial u_{j}} & k_{i j}=\frac{\partial P_{t}}{\partial E_{J}}
\end{array}\right]
$$

In expanded form it looks like

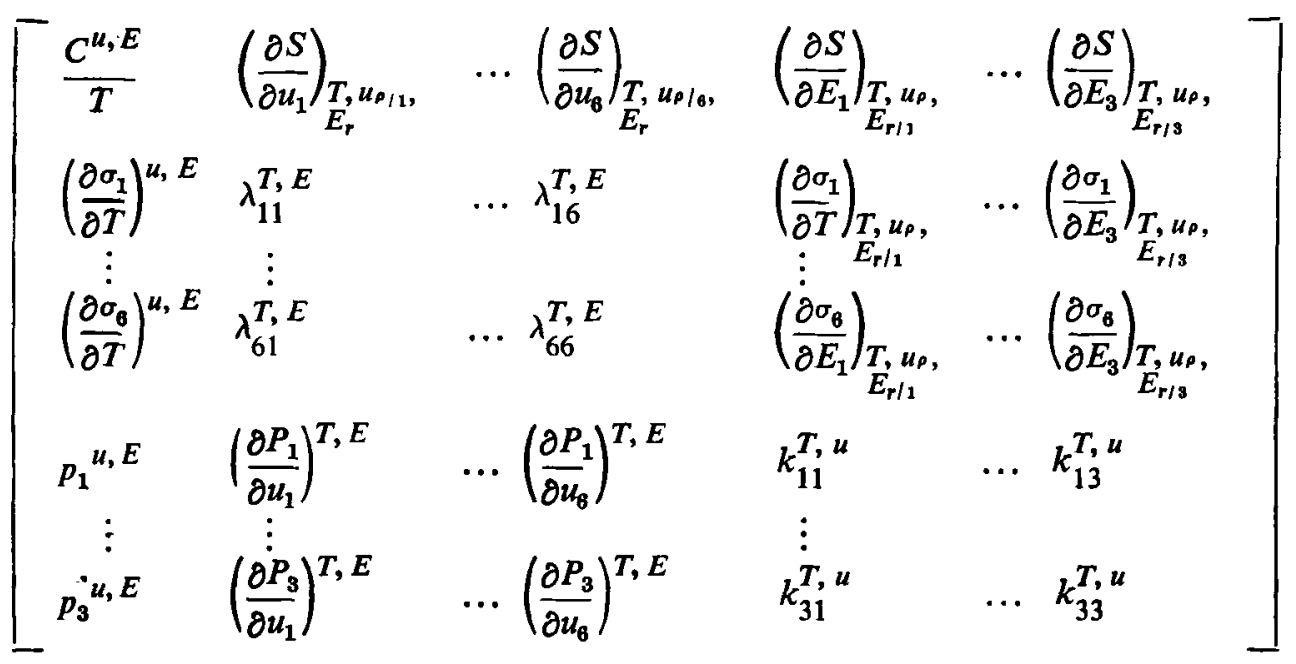

where $\quad C^{u, E} \quad$ is the heat capacity at constant strain and electric field,

$\lambda_{i j}^{T, E} \quad$ are the elastic coefficients at constant electric field,

$k_{i j}^{T, u} \quad$ are the isothermal dielectric susceptibilities at constant strain

$p_{i}^{u, E} \quad$ are the pyroelectric coefficients at constant strain and electric field.

The symbol $\rho / \mu$ denotes $\rho=1,2, \ldots, 6$.

In order to find the relation between the matrix components, we introduce the new variable.

$$
t=T-T_{c}\left(u_{\rho}, E_{r}\right)
$$




$$
\begin{aligned}
& \text { Now } \lambda_{\mu \nu}^{T, E}=\left(\frac{\partial \sigma_{\mu}}{\partial u_{\nu}}\right)_{T, u_{\rho} / \nu, E_{r}}=\frac{\partial\left(\sigma_{\mu}, T, u_{\rho / \nu}, E_{r}\right)}{\partial\left(u_{\nu}, T, u_{\rho / \nu}, E_{r}\right)} \\
& =\left[\frac{\partial\left(\sigma_{\mu}, T\right)}{\partial\left(u_{\nu}, T\right)}\right]_{u_{\rho / \nu}, E_{r}}\left(\frac{\partial t}{\partial T}\right)_{u_{\rho}, E_{\mathrm{r}}} \\
& =\left|\begin{array}{l}
\left(\frac{\partial \sigma_{\mu}}{\partial u_{\nu}}\right)_{t} \frac{\partial \sigma_{\mu}}{\partial t} \\
\left(\frac{\partial T}{\partial u_{\nu}}\right)_{t} \frac{\partial T}{\partial t}
\end{array}\right| \begin{array}{l}
\left(\frac{\partial t}{\partial T}\right)_{u_{\rho}, E_{r}} \\
u_{\rho / \nu}, E_{r}
\end{array} \\
& =\left(\frac{\partial \sigma_{\mu}}{\partial u_{\nu}}\right)_{t, u_{\rho / \nu}, E_{\mathrm{r}}}-\left(\frac{\partial \sigma_{\mu}}{\partial T}\right)_{u_{\rho}, E_{\mathrm{r}}}\left(\frac{\partial T}{\partial u_{\nu}}\right)_{t, u_{\rho} / v, E_{\mathrm{r}}} \\
& =\lambda_{\mu \nu}^{t, E}-\left(\frac{\partial S}{\partial u}\right)_{T, u_{\rho / \nu}, E_{\mathrm{r}}}\left(\frac{\partial T}{\partial u_{\nu}}\right)_{t, u_{\rho / \nu} E_{\mathrm{r}}}
\end{aligned}
$$

Similarly

$$
\left(\frac{\partial S}{\partial u}\right)_{T, u_{\rho} / \nu, E_{\mathrm{r}}}=\left(\frac{\partial S}{\partial u}\right)_{t, u_{\rho / v}, E_{\mathrm{r}}}-\left(\frac{\partial T}{\partial u_{\nu}}\right)_{t, u_{\rho} / v, E_{r}}\left(\frac{C^{u, E}}{T}\right)
$$

From (1) and (2)

$$
\begin{aligned}
\lambda_{\mu \nu}^{T, E} & =\lambda_{\mu \nu}^{t, E}-\left[\left(\frac{\partial S}{\partial u}\right)_{t, u_{\rho / \nu}, E_{r}}-\left(\frac{\partial T}{\partial u_{\nu}}\right)_{t, u_{\rho / \nu}, E_{r}}\left(\frac{C u, E}{T}\right)\right]\left(\frac{\partial T}{\partial u_{\nu}}\right)_{t, u \rho, \nu,} E_{r} \\
& =\left(\frac{\partial T}{\partial u_{\nu}}\right)_{t, u_{\rho / \nu}, E_{r}}^{2} \frac{C u, E}{T}+\text { constant. }
\end{aligned}
$$

It should be noted that this is a relation between the elastic coefficient at constant temperature and specific heat at constant mechanical strain whereas experimentally measured quantities are elastic coefficient at constant entropy and the specific heat at constant mechanical stress. But as it will be shown below the above relation is valid for the experimentally measured quantities too. The isothermal and adiabatic elastic coefficients are related as follows (Nozdrev 1954).

$$
\lambda_{i j}^{T}=\lambda_{i j}^{S}-\frac{C_{v}}{\rho C_{p}} \lambda_{i j}^{S} a_{i j}^{2} \frac{\lambda_{i j}^{S} T}{C_{p}}
$$

The specific heat at constant volume is related to the specific heat at constant pressure in the following way (Nozdrev 1954)

$$
\frac{C_{v}}{T}=\frac{C_{p}}{T}-\frac{C_{v}}{\rho C_{p}} \lambda_{i j}^{S} a_{i j}^{2}
$$


From (4) and (5)

$$
\begin{aligned}
\frac{\lambda_{i j}^{T}}{\frac{C_{v}}{T}}= & \frac{\lambda_{i j}^{S}-\frac{C_{v}}{\rho C_{p}} \lambda_{i j}^{S} a_{i j}^{2} \frac{\lambda_{i j}^{S} T}{C_{p}}}{\frac{C_{p}}{T}-\frac{C_{v}}{\rho C_{p}} \lambda_{i j}^{S} \alpha_{i j}^{2}} \\
= & \frac{\lambda_{i j}^{S}\left(1-\frac{C_{v}}{\rho C_{p}} \lambda_{i j}^{S} a_{i j}^{2} \frac{T}{C_{p}}\right)}{\frac{C_{p}}{T}\left(1-\frac{C_{v}}{\rho C_{p}} \lambda_{i j}^{S} a_{i j}^{2} \frac{T}{C_{p}}\right)} \\
= & \frac{\lambda_{i j}^{S}}{C_{p}}
\end{aligned}
$$

We see $C_{v}, C_{p}$ are nothing but our $C^{u}$ and $C^{\sigma}$. Therefore from (3) and (6) we get

$$
\lambda_{i j}^{S}=\left(\frac{\partial T}{\partial u_{v}}\right)_{t, u_{\rho / \nu}, E_{\mathrm{r}}}^{2} \frac{C^{\sigma, E}}{T}+\text { constant. }
$$

This is an equation of a straight line. This relation can be used for correlating the anomaly of ultrasonic velocity with the anomaly of specific heat of ferroelectric triglycine selenate crystal (Khanna 1976). Figure 1 shows the dependence of the anomalous elastic coefficient on the specific heat of TGSe for the same temperature differences. It is clear that the points lie on a straight line. Finally one notes that Pippard-Janovec relations provide a good tool for testing the consistency of acoustical and thermal data.

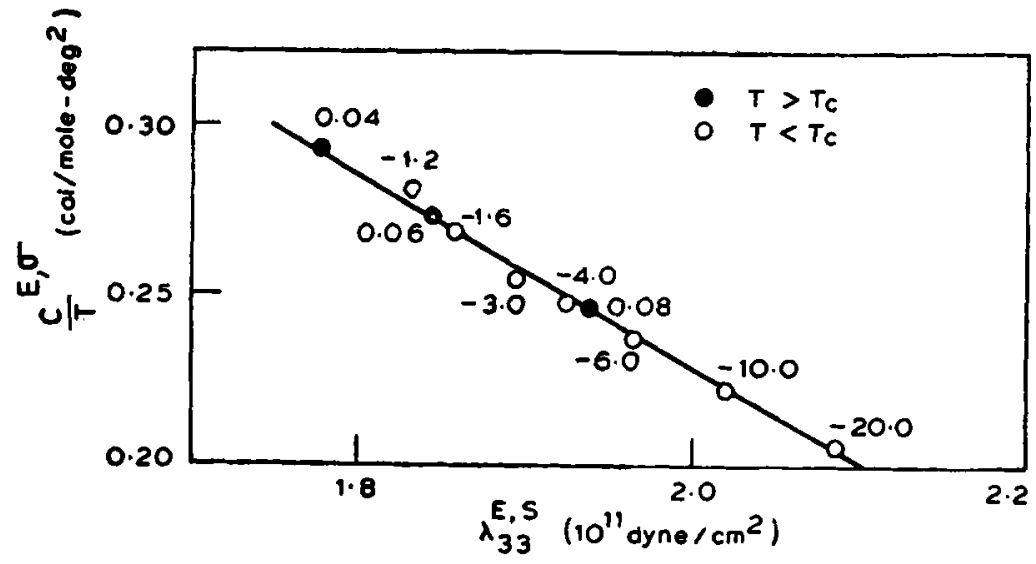

Figure 1. The dependence of the specific heat divided by temperature on the magnitude of the elastic coefficient $\lambda_{33}^{E, S}$ for TGSe crystal. 


\section{Acknowledgement}

One of the authors (SKK) is highly indebted to B A Strukov and K A Minaeva of Moscow State University for helpful discussions and suggestions.

\section{References}

Buckingham M J and Fairbank W M 1961 Prog. Low Temp. Phys. 380

Garland C W 1964 J. Chem. Phys. 411005

Garland C W 1970 in Physical Acoustics ed. W P Mason and R N Thurston (New York: Academic Press) Vol. 7 Ch. 2

Hughes A J and Lawson A W 1962 J. Chem. Phys. 362098

Janovec V 1966 J. Chem. Phys. 451874

Khanna S K 1976 Investigation of Critical Anomalies of Ultrasonic Velocity and Ultrasonic Absorption in Uniaxial Ferroelectric Crystals by Optical Heterodyne Method Ph.D. Thesis, Moscow State University, Moscow

Nozdrev V F and Fedorishenko N V 1954 Molecular Acoustics p 150

Pippard A B 1956 Philos. Mag. 1473

Pippard A B 1957 The Elements of Classical Thermodynamics (London: Cambridge University Press) 